Pacific Journal of Mathematics

DIFFERENTIAL EQUATIONS ON ABSTRACT WIENER SPACE 


\section{DIFFERENTIAL EQUATIONS ON ABSTRACT WIENER SPACE}

\section{ANN PIECH}

The main purpose of this paper is to indicate a simple method by means of which the work of $L$. Gross concerning the Laplacian on an abstract Wiener space may be extended to a certain class of pure second order elliptic operators with constant coefficients. A short proof of uniqueness of the solution semigroup of the heat equation will also be given.

Our extension method is motivated by the often-used technique of performing a change of variables in order to reduce a pure second order elliptic operator on $R^{n}$ with constant coefficients to the Laplacian. However, some fundamental dissimilarities between finite dimensional and infinite dimensional potential theory must be taken into account. First let us define an infinite dimensional Laplacian. Let $H$ denote a real separable Hilbert space and $D^{2} f(x)$ denote the second Fréchet derivative of a real-valued function $f$ on $H$. We may regard $D^{2} f(x)$ as a bounded linear operator on $H$. We define $\Delta f(x) \equiv \operatorname{trace} D^{2} f(x)$ whenever $D^{2} f(x)$ exists and is of trace class. This obviously extends the finite dimensional Laplacian. However, unlike the finite dimensional case, the existence of $D^{2} f(x)$ is not sufficient to ensure the existence of $\Delta f(x)$. Another dissimilarity is a consequence of the unavailability of a substitute for $n$-dimensional Lebesgue measure which would be countably additive on the Borel field of $H$. Use of Gauss cylinder set measure can provide an integration theory on $H$, but this is not adequate for potential theory, and more particularly for regularity studies. The reason for this inadequacy is that a Brownian motion defined in $H$ in terms of Gauss cylinder set measure would have the property that the probability of a particle starting at the origin and instantly leaving the ball of radius $r>0$ would be one.

To avoid this inadequacy, the concept of an abstract Wiener space $(H, B, i)$ was introduced by Gross [1]. $B$ denotes the completion of $H$ with respect to a fixed measurable norm $\|\cdot\|$, and $i$ is the natural injection of $H$ into $B$. Gauss cylinder set measure on $H$ determines a cylinder set measure on $B$, which in turn extends to a countably additive Borel measure on $B$ (Wiener measure). The measure on $B$ determined by Gauss measure on $H$ with variance parameter $t>0$ is denoted by $p_{t}$. For a Borel set $\Gamma \subset B$ and $x \in B$, let $p_{t}(x, \Gamma) \equiv$ $p_{t}(\Gamma-x)$. The measures $p_{t}(x, \cdot)$ give the transition probabilities 
for a Wiener process with continuous sample paths initiating at the origin of $B$.

Problems in potential theory are stated in terms of a fixed $(H$, $B, i)$. If $u(t, x)$ is a real valued function on $[0, \infty) \times B$ and if

$$
\left(t_{0}, x_{0}\right) \in[0, \infty) \times B
$$

is fixed, then we may consider $h(x) \equiv u\left(t_{0}, x_{0}+x\right)$ as a function from $H$ into $R$. The second $H$-derivative of $u$ at $\left(t_{0}, x_{0}\right)$ is defined as

$$
D^{2} u\left(t_{0}, x_{0}\right) \equiv D^{2} h(0) \text {. }
$$

The initial value problem for the heat equation can now be stated as

$$
\begin{aligned}
\frac{\partial}{\partial t} u(t, x) & =\operatorname{trace} D^{2} u(t, x) \\
u(0, x) & =f(x)
\end{aligned}
$$

where $x$ varies over $B$. We note that we are only concerned with differentiation in directions of $H$, even though the space variable ranges over $B$. In an analogous fashion, open sets in $B$ are appropriate for a statement of the Dirichlet problem.

Let $A$ be a fixed member of $L(H)$ (the space of bounded linear operators on $H$ ) satisfying

(a- i ) $A$ is symmetric,

(a-ii) $A \geqq \varepsilon I$ for some $\varepsilon>0$,

(a-iii) $A=I+C$ where $C$ is of Hilbert-Schmidt class.

We claim that within the context of a given abstract Wiener space $(H, B, i)$ most of the results of Ref. [2] hold when the Laplacian is replaced by the differential operator trace $A D^{2} f(x)$.

Properties (a-i) and (a-ii) guarantee that $\sqrt{A}$ exists as a positive symmetric invertible member of $L(H)$. When $H$ is finite dimensional it is customary to transform trace $A D^{2} f(x)$ into the Laplacian of $f$ by making the change of variables $x \rightarrow \sqrt{A}-1$. Now $H=B$ when $H$ is finite dimensional; otherwise $H \subsetneq B$. Since $x$ is to vary over $B$, this application of a change of variables is meaningless for infinite dimensional $H$. It turns out that, rather than transforming the differential operator, we can meaningfully transform the fundamental solution of the heat equation.

Let $H_{A}$ be the Hilbert space obtained by replacing the inner product (, ) on $H$ by $[h, k] \equiv(\sqrt{A}-1 h, \sqrt{A}-1 k)$. The invertibility of $\sqrt{A} \bar{A}^{-1}$ ensures that [,] and (, ) give rise to equivalent norms. Thus $\|\cdot\|$ is a measurable norm on $H_{A}$, and we may also view $B$ as the completion of $H_{A}$ with respect to $\|\cdot\|$. If $i_{A}$ denotes the natural injection of $H_{A}$ into $B$, then $\left(H_{A}, B, i_{A}\right)$ is an abstract Wiener space. We 
will let $p_{t}^{A}$ denote that measure on $B$ determined by Gauss cylinder set measure on $H_{A}$ with variance parameter $t>0 . \quad p_{t}^{A}$ will be called Wiener measure on $\left(H_{A}, B, i_{A}\right)$.

Wiener measure $p_{t}$ on $(H, B, i)$ gives rise to a fundamental solution of the heat equation

$$
\frac{\partial}{\partial t} u(t, x)=\operatorname{trace} D^{2} u(t, x)
$$

( $x$ ranges over $B$, and $t$ over $(0, \infty)$ ). Specifically, the family

$$
\left\{p_{2 t}(x, d y): x \in B, t>0\right\}
$$

has the following properties [2, Theorem 3 and Porposition 6]:

For each bounded real-valued uniformly Lip 1 function $f$ on $B$, letting

$$
p_{t} f(x) \equiv \int_{B} f(y) p_{t}(x, d y),
$$

(b-i) $\quad p_{2 t} f(x)$ satisfies the heat equation (1)-that is, $\sigma /(\sigma t) p_{2 t} f(x)$ and $D^{2} p_{2 t} f(x)$ exist, $D^{2} p_{2 t} f(x)$ is of trace class and the equality (1) holds;

(b-ii) $\quad p_{2 t} f(x) \rightarrow f(x)$ as $t \downarrow 0$, uniformly for all $x$ in $B$. As a consequence of (b-i) and (b-ii), we say that

$$
\left\{p_{2 t}(x, d y) ; x \in B, t>0\right\}
$$

forms a fundamental solution of the heat equation.

By analogy with the finite dimensional situation, we expect the measures $\left\{q_{t}(x, d y): x \in B, t>0\right\}$ defined by

$$
q_{t}(x, d y) \equiv[\operatorname{det} A]^{-1 / 2} e^{-\left(\left[A^{-1}-I\right](x-y), x-y\right) / 4 t} p_{2 t}(x, d y)
$$

to form a fundamental solution of

$$
\frac{\partial}{\partial t} u(t, x)=\operatorname{trace} A D^{2} u(t, x) \text {. }
$$

That is, we expect that for each $f$ in the family $\mathscr{A}$ of bounded real-valued uniformly Lip 1 functions on $B$, the function

$$
q_{t} f(x) \equiv \int_{B} f(y) q_{t}(x, d y)
$$

satisfies (3) and $q_{t} f \rightarrow f$ in sup norm as $t \downarrow 0$.

REMARK. We must explain the meaning of the exponential term which occurs in the expression for $q_{t}(x, d y)$. It is to be interpreted 
as the limit in mean $\left(p_{2 t}(x, d y)\right)$ as $F \rightarrow I$ of the net of tame functions $\left\{\exp \left[-\left(\left[A^{-1}-I\right]\left(x-P_{F} y\right), x-P_{F} y\right) / 4 t\right]: F\right.$ is a finite dimensional subspace of $H$ which is left invariant by $C$ and $P_{F}$ is projection onto $F$ \}. The integral of a tame function with respect to $p_{t}$ is described in Ref. [1]. We will see later that this net does converge.

A direct verification that $\left\{q_{t}(x, d y)\right\}$ has the properties of a fundamental solution would be both difficult and lengthy. However, Theorems 2 and 3 of Ref. [5] assert that $p_{t}^{A}$ is mutually absolutely continuous with respect to $p_{t}$, with Radon-Nikodym derivative given by

$$
p_{t}^{A}(d y)=[\operatorname{det} A]^{-1 / 2} e^{-\left(\left[A^{-1-I] y, y) / 2 t}\right.\right.} p_{t}(d y),
$$

provided $\sqrt{A}-I$ is of Hilbert-Schmidt class. The latter property is verified by writing $\sqrt{A}=I+C[I+\sqrt{A}]^{-1}$. Setting $p_{t}^{A}(x, \Gamma) \equiv$ $p_{t}^{A}(\Gamma-x)$ for Borel sets $\Gamma$ in $B$, we see that $q_{t}(x, d y)=p_{2 t}^{A}(x, d y)$. We may now appeal to the work of Gross [2] to establish many properties of $\left\{q_{t}(x, d y)\right\}$. Before doing so, however, we recall some properties of trace class operators.

We will identify individual elements of $H$ and of $H_{A}$ via the identity map on the topological vector space $H$. Similarly we will identity individual elements of $L(H)$ and $L\left(H_{A}\right)$. We recall that the family of trace class operators in $L(H)$ is

$\left\{T \in L(H): \sum_{i=1}^{\infty}\left(\left[T^{*} T\right]^{1 / 2} e_{i}, e_{i}\right)<\infty\right.$ for some orthonormal basis $\left\{e_{i}\right\}$ of $\left.H\right\}$,

with the trace of $T$ defined as $\operatorname{Tr} T \equiv \sum_{i=1}^{\infty}\left(T e_{i}, e_{i}\right)$ where $\left\{e_{i}\right\}$ is any orthonormal basis of $H$. The trace class norm of $T \in L(H)$ is

$$
|T|_{\operatorname{Tr} L(H)} \equiv \operatorname{Tr}\left[T^{*} T\right]^{1 / 2} \text {. }
$$

The completely continuous operators in $L(H)$ with $|\cdot|_{L(H)}$ form the dual of the space of trace class operators in $L(H)$ under the pairing $\langle U, V\rangle \equiv \operatorname{Tr} U^{*} V$, where $U$ is completely continuous and $V$ is of trace class. Since operators of finite rank are dense in the space of completely continuous operators, we may write $|T|_{\operatorname{Tr} L(H)}=$ $\sup \left\{|\operatorname{Tr}[T F]| /|F|_{L(H)}: F\right.$ is of finite rank in $L(H)$ and $\left.F \neq \equiv 0\right\}$. For any $S$ in $L(H)$ and $T$ of trace class, $S T$ and $T S$ are of trace class and $\operatorname{Tr} S T=\operatorname{Tr} T S$. Thus the set of trace class operators on $H$ is invariant under a change of inner product. Consequently the set of trace class operators and their traces are the same whether we consider $L(H)$ or $L\left(H_{A}\right)$. The trace class norm does vary with the change of inner product, although $|\cdot|_{\operatorname{Tr} H}$ and $|\cdot|_{\operatorname{Tr}_{A}}$ are equivalent norms.

We point out that, by definition, $D^{2} q_{t} f(x)$ is a member of $L(H$, $\left.H^{*}\right)$. The identification of $D^{2} q_{t} f(x)$ with an element of $L(H)$ is 
dependent on the inner product assigned to $H$. Unless otherwise specified, we will always intend this identification to be via (,). If we let $T(t, x)$ denote the operator in $L(H)$ determined by considering $D^{2} q_{t} f(x)$ as a member of $L\left(H_{A}, H_{A}^{*}\right)$, identifying $H_{A}^{*}$ with $H_{A}$ via [, ] and $L\left(H_{A}\right)$ with $L(H)$, then $T(t, x)=A D^{2} q_{t} f(x)$. Since

$$
\left\{p_{2 t}^{A}(x, d y): x \in B, t>0\right\}
$$

is a fundamental solution of the heat equation in $\left(H_{A}, B, i_{A}\right)$, we immediately have the

Proposition 1. Assume $A$ satisfies (a-i), (a-ii) and (a-iii). Then $\left\{q_{t}(x, d y): x \in B, t>0\right\}$ forms a fundamental solution of the equation

$$
\frac{\partial}{\partial t} u(t, x)=\operatorname{trace} A D^{2} u(t, x) \text {. }
$$

REMARK. The existence of fundamental solutions of Eq. (3) in situations where $A$ is nonconstant has been considered by the author in [3]. There $A-I$ was assumed to be of trace class, and this property was relied upon considerably. Proposition 1 allows generalization of the results of Ref. [3] to situations where $A$ is of the form $I+C_{1}+C_{2}$ where $A \geqq \varepsilon I$ for some $\varepsilon>0, I+C_{1}$ satisfies (a-i)-(a-iii) and $I+C_{2}$ satisfies the hypotheses made in Ref. [3]. Generally speaking, then, such an $A$ is of the form identity plus a constant Hilbert-Schmidt class operator plus a variable trace class operator. We conjecture that the results of Ref. [3] may be extended to operators of the form identity plus a variable Hilbert-Schmidt class operator.

Now let us assume that $f \in \mathscr{A}$ and that $f$ has bounded support. We may apply the preceding technique to obtain a solution of

$$
\text { trace } A D^{2} u(x)=f(x) \text {. }
$$

We define the Green's measures $G$ and $G_{A}$ on Borel sets $\Gamma$ of $B$ by

$$
G(\Gamma) \equiv \int_{0}^{\infty} p_{t}(\Gamma) d t
$$

and

$$
G_{A}(\Gamma) \equiv \int_{0}^{\infty} q_{t}(\Gamma) d t,
$$

and the potentials $G h$ and $G_{A} h$ of a Borel function $h$ on $B$ by

$$
G h(x) \equiv \int_{B} h(x+y) G(d y)
$$

and 


$$
G_{A} h(x) \equiv \int_{B} h(x+y) G_{A}(d y) .
$$

Then by Ref. [2, Theorem 3], $G f(x)$ satisfies

$$
\frac{1}{2} \operatorname{trace}\left[\left(D^{2} G f\right)(x)\right]=-f(x)
$$

for all $x$ in $B$. We thus immediately have the

Proposition 2. Assume $A$ satisfies (a-i), (a-ii) and (a-iii). For $f$ in $\mathscr{A}$ and of bounded support,

$$
u(x) \equiv-G_{A} f(x)
$$

satisfies Eq. (4).

REMARK. Many smoothness properties and corresponding estimates concerning $p_{t} f(x)$ and $G f(x)$ are given in Ref. [2]. Analogues of these may now trivially be deduced for $q_{t} f(x)$ and $G_{A} f(x)$.

From Ref. [2] we see that for $t>0$ the operators $q_{t}: f \rightarrow q_{t} f$ form a strongly continuous contraction semigroup on the space $\mathscr{C}$ of bounded uniformly continuous functions $f$ on $B$ with $\|f\|_{\infty}$. Let $\mathscr{L}$ denote the infinitesimal generator of this semigroup. Then [2, Cor. 3.1] for $f$ in $\mathscr{A}, q_{t} f$ is in the domain $\mathscr{D}_{\mathscr{S}}$ of $\mathscr{L}$ and

$$
\left(\mathscr{L} q_{t} f\right)(x)=\operatorname{trace}\left[\left(A D^{2} q_{t} f\right)(x)\right] \equiv L f(x) .
$$

A question naturally arises concerning possible uniqueness of the semigroup $\left\{q_{t}: t>0\right\}$ among semigroups on $\mathscr{C}$ whose infinitesimal generators are "related" to $L$. This question for variable coefficients $A(x)$ will be discussed by the author in a forthcoming paper [4]. The method used there could be applied to the case presently under consideration. However Ref. [4] makes use of a theory of stochastic integrals on $(H, B, i)$, which requires a special hypothesis on the abstract Wiener space $(H, B, i)$. Moreover, the approach of [4] is unduly cumbersome in the constant coefficient case. Therefore we will now present a brief uniqueness result for the constant coefficient case. We begin by showing that $\mathscr{P}$ is the closure of $L$. Specifically, we have the

Proposition 3. Let the set $\mathscr{S}$ consist of real-valued functions $f$ satisfying

(c-i ) $f$ is in $\mathscr{A}$;

(c-ii) $D f: B \rightarrow H$ exists, is bounded and continuous;

(c-iii) $D^{2} f: B \rightarrow$ trace class operators on $H$ with $|\cdot|_{\operatorname{Tr} H}$ exists, is bounded and uniformly continuous. 
Then $\mathscr{S} \subset \mathscr{D}_{\mathscr{L}}$, for $f$ in $\mathscr{S} \mathscr{L} f=L f$, and $\{(f, \mathscr{L} f): f \in \mathscr{S}\}$ is dense in the closed subset $\left\{(f, \mathscr{L} f): f \in \mathscr{D}_{\mathscr{L}}\right\}$ of $\mathscr{C} \times \mathscr{C}$.

Proof. Assume $f$ is in $\mathscr{S}$. Since

$$
\left(q_{t} f\right)(x+s h)=\int_{B} f(x+s h+y) q_{t}(d y),
$$

(c-ii) enables differentiation under the integral sign, yielding

$$
\left(D q_{t} f(x), h\right)=\int_{B}(D f(x+y), h) q_{t}(d y)
$$

for all $h$ in $H$. Similarly (c-iii) enables us to write

$$
\left(\left(D^{2} q_{t} f\right)(x) k, h\right)=\int_{B}\left(D^{2} f(x+y) k, h\right) q_{t}(d y)
$$

for all $k$ and $h$ in $H$, and

$$
L q_{t} f(x)=\int_{B} L f(x+y) q_{t}(d y) .
$$

Since $q_{t} f \in \mathscr{D}_{\mathscr{f}}$, we have

$$
\mathscr{L} q_{t} f=q_{t} L f .
$$

$L f$ is in $\mathscr{C}$ by (c-iii). Thus $q_{t} L f \rightarrow L f$ uniformly as $t \downarrow 0$, and so $\mathscr{L} q_{t} f \rightarrow L f$ uniformly as $t \downarrow 0$. But $q_{t} f \rightarrow f$ uniformly as $t \downarrow 0$ and, since $\mathscr{L}$ is a closed operator by basic semigroup theory, we conclude that $f$ is in $\mathscr{D}_{\mathscr{e}}$ and $\mathscr{L} f=L f$.

It is shown in Ref. [2, Cor. 3.2] that functions of the form

$$
g(x)=\int_{0}^{\infty} e^{-t}\left(p_{t} f\right)(x) d t
$$

where $f \in \mathscr{A}$ are dense in the domain of $\mathscr{L}$ in the graph $(\mathscr{C} \times \mathscr{C})$ norm. It is furthermore shown that such functions $g$ satisfy (c-iii). It is trivial to see that $q_{t}: \mathscr{A} \rightarrow \mathscr{A}$, and hence that $g \in \mathscr{A}$. To verify (c-ii) we make use of Eq. (8) of Ref. [2]-viz. for $h$ in $H$

$$
\left(D q_{t} f(x), h\right)=(2 t)^{-1} \int_{B} f(x+y)[h, y] q_{t}(d y) .
$$

Thus we obtain

$$
\begin{aligned}
(D g(x), h) & =\int_{0}^{\infty} e^{-t}(2 t)^{-1} \int_{B} f(x+y)[h, y] q_{t}(d y) d t \\
& =\int_{0}^{\infty} e^{-t}(2 t)^{-1 / 2} \int_{B} f\left(x+(2 t)^{1 / 2} y\right)[h, y] p_{1}^{A}(d y) d t
\end{aligned}
$$

Therefore 


$$
\begin{aligned}
|(D g(x), h)| & <\int_{0}^{\infty} e^{-t} t^{-1 / 2}\|f\|_{\infty}\left\{\int_{B}|[h, y]|^{2} p_{1}^{A}(d y)\right\}^{1 / 2} d t \\
& \leqq\|f\|_{\infty}|h|_{H_{A}} \int_{0}^{\infty} e^{-t} t^{-1 / 2} d t
\end{aligned}
$$

and so

$$
|D g(x)|_{H I} \leqq \text { constant } \cdot\|f\|_{\infty} \cdot
$$

In addition, we see that

$$
|(D g(x)-D g(z), h)| \leqq \text { constant } \cdot \int_{0}^{\infty} e^{-t} t^{-1 / 2}|| x-z \|\left.|| h\right|_{I I} d t,
$$

and we conclude that $g$ satisfies (c-ii).

Thus we have proved that $\left(\mathscr{L}, \mathscr{D}_{\mathscr{C}}\right)$ is the closure of $(L, \mathscr{S})$.

REMARK. The preceding calculations of $D q_{t} f$ and $D^{2} q_{t} f$ were possible because $q_{t} f$ is a convolution of $f$ with $p_{t}(d y)$. This is not the case with variable coefficients.

We now give a uniqueness result for the semigroup $\left\{q_{t}\right\}$.

Proposition 4. If $\left\{q_{t}^{\prime}: t>0\right\}$ is a contraction semigroup on $\mathscr{C}$ whose infinitesimal generator $\mathscr{L}^{\prime}$ extends $(L, \mathscr{S})$, then $q_{t}^{\prime}=q_{t}$ for all $t>0$.

Proof. If we show that $\mathscr{D}_{\mathscr{N}}=\mathscr{D}_{\mathscr{C}}$ and that $\mathscr{L}^{\prime}=\mathscr{C}$ on their common domain, then since $\left\{q_{t}\right\}$ is strongly continuous on $\mathscr{C}$ it follows from basic semigroup theory that $q_{t}^{\prime}=q_{t}$. Since $\left(\mathscr{P}^{\prime}, \mathscr{D}_{-}^{\prime}\right)$ is a closed operator, we have $\left(\mathscr{L}^{\prime}, \mathscr{D}\right) \supset\left(\mathscr{L}, \mathscr{D}_{\mathscr{C}}\right)$. Let

$$
f \in \mathscr{D}_{\varkappa \prime}, g \equiv\left(I-\mathscr{L}^{\prime}\right) f
$$

and

$$
\mathscr{C}_{0} \equiv\left\{h \text { in } \mathscr{C}:\left\|q_{t}^{\prime} h-h\right\|_{\infty} \rightarrow 0 \text { as } t \downarrow 0\right\} \text {. }
$$

$\mathscr{D}_{-}, \subset \mathscr{C}_{0}$ and $\mathscr{L}^{\prime}: \mathscr{C}_{0} \rightarrow \mathscr{C}_{0}$. Thus $g \in \mathscr{C}_{0}$. It is well known that for $g$ in $\mathscr{C}_{0}$ the equation $\left(I-\mathscr{C}^{\prime}\right) h=g$ has a unique solution in $\mathscr{D} \ldots$ By the strong continuity of $\left\{q_{t}\right\}$, there exists a unique solution $\tilde{f}$ in $\mathscr{D}_{\mathscr{D}}$ of the equation $(I-\mathscr{L}) h=g$. Since $\tilde{f}$ is also in $\mathscr{D} \ldots, \mathscr{L}^{\prime} \tilde{f}=\mathscr{L} \tilde{f}$ and so $\tilde{f}=f$. Thus $\left(\mathscr{C}^{\prime}, \mathscr{D}_{\mathscr{L}}^{\prime}\right) \subset\left(\mathscr{L}, \mathscr{D}_{\mathscr{L}}\right)$.

\section{REFERENCES}

1. L. Gross, Abstract Wiener spaces, Proc. Fifth Berkeley Symposium on Math. Statistics and Probability, (1965), 31-42.

2. — Potential theory on Hilbert space, J. Functional Analysis, 1 (1967), 123181. 
3. M. A. Piech, A fundamental solution of the parabolic equation on Hilbert space, J. Functional Analysis, 3 (1969), 85-114.

4. - Diffusion semigroups on abstract Wiener space, Trans. Amer. Math. Soc., 166 (1972), 411-429.

5. I. E. Segal, Distributions in Hilbert space and canonical systems of operators, Trans. Amer. Math. Soc., 88 (1958), 12-41.

Received August 16, 1971. This work was supported by NSF grants GP-19651 and GP-28934.

State University of New York at Buffalo 



\section{PACIFIC JOURNAL OF MATHEMATICS}

\section{EDITORS}

\author{
H. SAMELSON \\ Stanford University \\ Stanford, California 94305 \\ C. R. HOBBY \\ University of Washington \\ Seattle, Washington 98105
}

\author{
J. DugundJI \\ Department of Mathematics \\ University of Southern California \\ Los Angeles, California 90007
}

\author{
RICHARD ARENS \\ University of California \\ Los Angeles, California 90024
}

\section{ASSOCIATE EDITORS}
E. F. BECKENBACH
B. H. NeumanN
F. WOLF
K. YoSHIDA

\section{SUPPORTING INSTITUTIONS}

\author{
UNIVERSITY OF BRITISH COLUMBIA \\ CALIFORNIA INSTITUTE OF TECHNOLOGY \\ UNIVERSITY OF CALIFORNIA \\ MONTANA STATE UNIVERSITY \\ UNIVERSITY OF NEVADA \\ NEW MEXICO STATE UNIVERSITY \\ OREGON STATE UNIVERSITY \\ UNIVERSITY OF OREGON \\ OSAKA UNIVERSITY
}

\author{
UNIVERSITY OF SOUTHERN CALIFORNIA \\ STANFORD UNIVERSITY \\ UNIVERSITY OF TOKYO \\ UNIVERSITY OF UTAH \\ WASHINGTON STATE UNIVERSITY \\ UNIVERSITY OF WASHINGTON \\ $* * * *$
$*$
AMERICAN MATHEMATICAL SOCIETY
NAVAL WEAPONS CENTER
}

The Supporting Institutions listed above contribute to the cost of publication of this Journal, but they are not owners or publishers and have no responsibility for its content or policies.

Mathematical papers intended for publication in the Pacific Journal of Mathematics should be in typed form or offset-reproduced, (not dittoed), double spaced with large margins. Underline Greek letters in red, German in green, and script in blue. The first paragraph or two must be capable of being used separately as a synopsis of the entire paper. The editorial "we" must not be used in the synopsis, and items of the bibliography should not be cited there unless absolutely necessary, in which case they must be identified by author and Journal, rather than by item number. Manuscripts, in duplicate if possible, may be sent to any one of the four editors. Please classify according to the scheme of Math. Rev. Index to Vol. 39. All other communications to the editors should be addressed to the managing editor, Richard Arens, University of California, Los Angeles, California, 90024.

50 reprints are provided free for each article; additional copies may be obtained at cost in multiples of 50 .

The Pacific Journal of Mathematics is published monthly. Effective with Volume 16 the price per volume (3 numbers) is $\$ 8.00$; single issues, $\$ 3.00$. Special price for current issues to individual faculty members of supporting institutions and to individual members of the American Mathematical Society: $\$ 4.00$ per volume; single issues $\$ 1.50$. Back numbers are available.

Subscriptions, orders for back numbers, and changes of address should be sent to Pacific Journal of Mathematics, 103 Highland Boulevard, Berkeley, California, 94708.

PUBLISHED BY PACIFIC JOURNAL OF MATHEMATICS, A NON-PROFIT CORPORATION

Printed at Kokusai Bunken Insatsusha (International Academic Printing Co., Ltd.), 270, 3-chome Totsuka-cho, Shinjuku-ku, Tokyo 160, Japan. 


\section{Pacific Journal of Mathematics}

\section{Vol. 43, No. 2 \\ April, 1972}

Arne P. Baartz and Gary Glenn Miller, Souslin's conjecture as a problem on the real line....................................... 277

Joseph Barback, On solutions in the regressive isols ............... 283

Barry H. Dayton, Homotopy and algebraic K-theory ................ 297

William Richard Derrick, Weighted convergence in length ............ 307

M. V. Deshpande and N. E. Joshi, Collectively compact and semi-compact sets of linear operators in topological vector spaces ............. 317

Samuel Ebenstein, Some $H^{p}$ spaces which are uncomplemented in $L^{p} \ldots . .327$

David Fremlin, On the completion of locally solid vector lattices ......... 341

Herbert Paul Halpern, Essential central spectrum and range for elements of

a von Neumann algebra............................... 349

G. D. Johnson, Superadditivity intervals and Boas' test ............. 381

Norman Lloyd Johnson, Derivation in infinite planes . . . . . . . . . . . 387

V. M. Klassen, The disappearing closed set property .............. 403

B. Kuttner and B. N. Sahney, On the absolute matrix summability of Fourier series ........................................... 407

George Maxwell, Algebras of normal matrices................... 421

Kelly Denis McKennon, Multipliers of type $(p, p) \ldots \ldots \ldots \ldots \ldots \ldots . . \ldots 29$

James Miller, Sequences of quasi-subordinate functions ............. 437

Leonhard Miller, The Hasse-Witt-matrix of special projective varieties ..... 443

Michael Cannon Mooney, A theorem on bounded analytic functions ...... 457

M. Ann Piech, Differential equations on abstract Wiener space .......... 465

Robert Piziak, Sesquilinear forms in infinite dimensions ............. 475

Muril Lynn Robertson, The equation $y^{\prime}(t)=F(t, y(g(t))) \ldots \ldots \ldots \ldots .483$

Leland Edward Rogers, Continua in which only semi-aposyndetic

subcontinua separate ............................... 493

Linda Preiss Rothschild, Bi-invariant pseudo-local operators on Lie

groups ...................................... 503

Raymond Earl Smithson and L. E. Ward, The fixed point property for

arcwise connected spaces: a correction ...................... 511

Linda Ruth Sons, Zeros of sums of series with Hadamard gaps .......... 515

Arne Stray, Interpolation sets for uniform algebras............... 525

Alessandro Figà-Talamanca and John Frederick Price, Applications of random Fourier series over compact groups to Fourier multipliers .. 